\title{
Strates
}

STRATES Matériaux pour la recherche en sciences sociales

14 | 2008

Espaces du quotidien

\section{Voie express et recomposition des territoires quotidiens entre Yvelines et Eure-et-Loir}

\section{Claire Aragau}

\section{(2) OpenEdition}

1 Journals

\section{Édition électronique}

URL : http://journals.openedition.org/strates/6670

DOI : 10.4000/strates.6670

ISSN : 1777-5442

\section{Éditeur}

Laboratoire Ladyss

\section{Édition imprimée}

Date de publication : 1 janvier 2008

Pagination : 135-148

ISBN : 0768-8067

ISSN : 0768-8067

\section{Référence électronique}

Claire Aragau, « Voie express et recomposition des territoires quotidiens entre Yvelines et Eure-et-Loir », Strates [En ligne], 14 | 2008, mis en ligne le 04 mars 2013, consulté le 08 septembre 2020. URL : http://journals.openedition.org/strates/6670; DOI : https://doi.org/10.4000/strates.6670

Ce document a été généré automatiquement le 8 septembre 2020

Tous droits réservés 


\title{
Voie express et recomposition des territoires quotidiens entre Yvelines et Eure-et-Loir
}

\author{
Claire Aragau
}

1 Les infrastructures de transport desservent des espaces et contribuent à l'organisation des territoires qu'elles traversent. Des études d'impact concernant des axes autoroutiers ont révélé qu'ils renforçaient souvent l'attractivité des pôles les plus importants plutôt que celle des centres urbains de second rang situés sur ces axes, en raison de la réduction de la distance-temps et d'un nombre limité d'échangeurs (Cohou, 2000; Ollivro, 1998; Observatoire A71, 1994). Qu'en est-il d'un autre type d'aménagement, une "voie express", offrant des caractéristiques bien différentes et notamment de nombreuses voies d'entrée et de sortie ? Dans l'ouest de l'île-de-France et aux portes de la région Centre, la N12, entre Bois-d'Arcy et Dreux, est devenue une voie express en 2001. Cette nouvelle voie a-t-elle accentué la polarisation du territoire par la ville nouvelle et entraîné le déclin des petites villes et des bourgs situés le long de l'axe? Quelles incidences a-t-elle eu sur la recomposition des territoires pratiqués par les habitants de ce secteur des Yvelines et de l'Eure-et-Loir?

2 L'examen des changements engagés par une infrastructure routière suscite des interrogations et nécessite une grande prudence dans la démarche à suivre. La méthodologie est souvent déficiente. L'« effet » séduit car il permet de fournir des idées simples, univoques. Les techniques traditionnelles d'évaluation des projets reposent sur des analyses coûts-avantages. Elles prennent en compte, si possible en les quantifiant et en les monétarisant, des effets, directs ou indirects. François Plassard rappelle que « la vision simpliste de mécanismes de cause à effet ne peut être conservée dès qu'on étudie les relations entre autoroute et développement régional» (Offner, 1993). Des interprétations erronées proviennent par exemple d'une comparaison hâtive entre un «avant» et un "après ». De plus ce que l'on peut faire avec un environnement physique n'est pas applicable à un environnement socio-économique: une «étude d'impact» peut être faite sur le milieu naturel mais il en va autrement pour 
l'environnement socio-économique. Adopter une démarche systémique permet de prendre en compte une conjonction de facteurs à un moment donné et d'échapper à une comparaison d'un «avant» et d'un «après » qui risquerait d'être erronée; comment comparer deux périodes qui ne pourront jamais l'être tant le contexte politique, économique et social a évolué ? En choisissant comme angle d'approche la N12 et ses transformations, celle-ci sera présentée comme agissant en interaction avec l'armature commerciale pour comprendre comment s'organisent des territoires de proximité autour de petites villes et de bourgs desservis par la voie express.

\section{Voie express et réseau des centres de commerces et de services}

3 La N12, voie express entre Bois-d'Arcy et Dreux, rapproche des pôles urbains qui pouvaient jusque-là être considérés comme éloignés. En raison des performances techniques de la route, de nouvelles proximités apparaissent. La distance-temps est réduite mais est aussi garantie par la fluidité de la circulation (absence de carrefours à niveau, interdiction des véhicules lents) et une limite supérieure de vitesse portée à 110 $\mathrm{km} / \mathrm{h}$. D'après l'enquête menée par la DDE préalablement à la DUP ${ }^{1}$ de 1992 , le gain de temps représenterait 6 minutes pour les véhicules légers et 8 minutes pour les poids lourds. Il faut noter que le gain est plus grand là où se trouvent d'anciens goulets d'étranglement, comme la traversée de Jouars-Pontchartrain. Le nombre des échangeurs, un tous les 2 à $3 \mathrm{~km}$, permet des connexions fréquentes avec les voies transversales. La N12 est incontestablement l'axe structurant le réseau routier. C'est autour de cette épine dorsale que s'articule le système viaire, incluant les routes nationales, départementales et communales. L'organisation en "peigne » permet une innervation efficace des villes de l'aire étudiée.

4 Ce réseau routier, dont le fonctionnement a été amélioré, dessert un ensemble de communes identifiées comme des pôles de commerces et de services (carte 1). L'inventaire communal de l'Insee permet de les classer par catégorie en fonction des types de services qu'ils proposent. Quatorze pôles relevant de la catégorie A de l'Insee ${ }^{2}$ sont recensés. Il s'agit du meilleur niveau pour les équipements essentiels. Cinq d'entre eux correspondent à des villes stricto sensu, les neuf autres sont plutôt des bourgs ruraux. Une hiérarchie s'établit entre ces pôles en fonction de leurs équipements et par conséquent de leur pouvoir d'attraction.

5 Les deux têtes du réseau sont les villes de Dreux et de Plaisir, situées chacune à une extrémité de l'axe N12. Elles comptent respectivement 31849 et 31045 habitants en 1999. Le nombre d'équipements donné par l'Insee pour chaque pôle permet de le positionner dans la hiérarchie urbaine. Il s'agit du nombre de commerces et de services dont dispose la commune parmi une série de 36. Dreux en détient 36 et Plaisir 34. Entre ces deux pôles majeurs se répartissent trois villes, de taille bien inférieure, mais ayant un niveau d'équipement encore élevé chiffré à 32. Il s'agit de Nogent-le-Roi en Eure-etLoir, de Houdan et de Montfort-l'Amaury dans les Yvelines, comptant 3000 à 4000 habitants en 1999. Ils sont distants en moyenne de 15 à $20 \mathrm{~km}$ et leur rayonnement est bien distinct. La troisième catégorie de ville ou bourg a des fonctions de moindre importance : entre 21 et 24 équipements. 


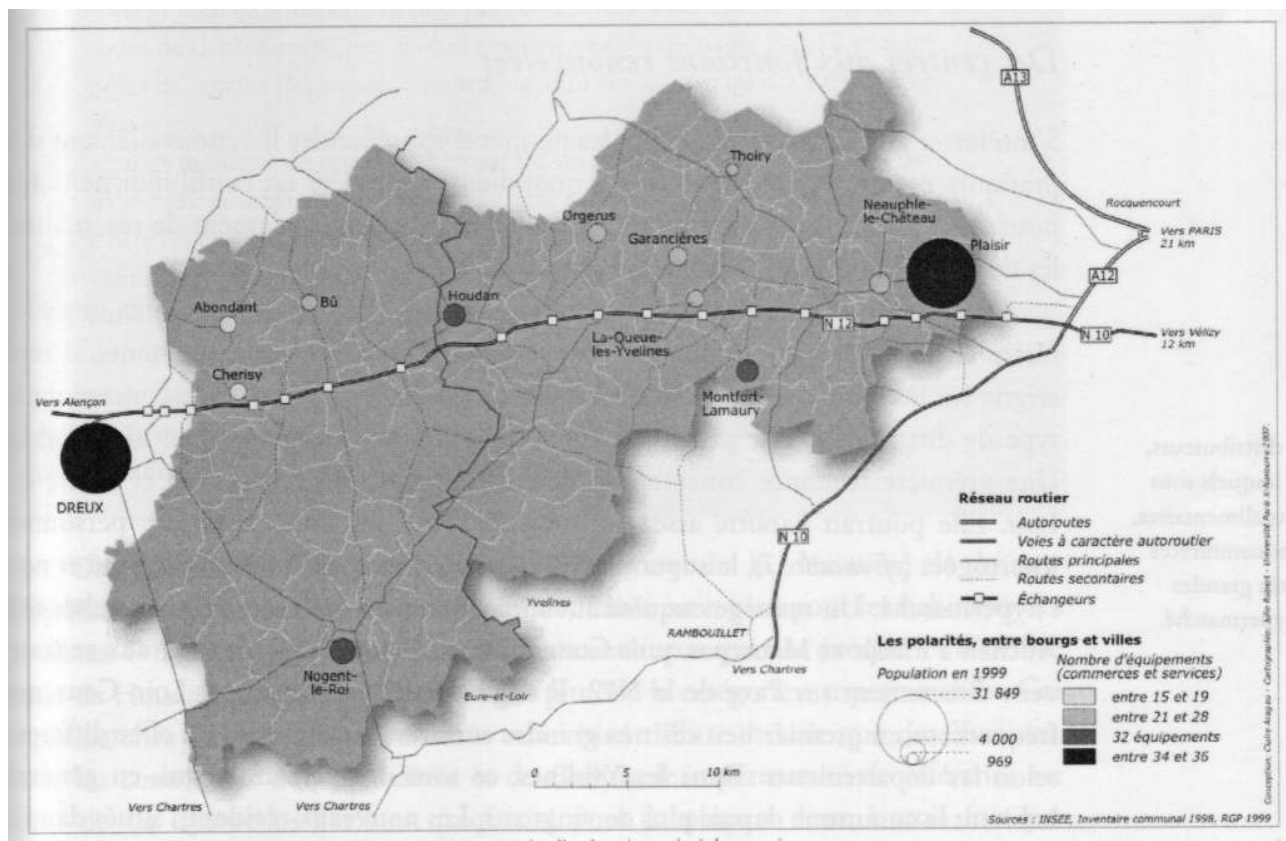

Plus nombreux côté Yvelines, ces pôles sont Neauphle-le-Château, La-QueuelesYvelines, Garancières, Thoiry et Orgerus. En Eure-et-Loir, seule Bû intègre ce niveau de classement dans la hiérarchie urbaine. Les plus petits pôles relevant de la catégorie A ont un nombre d'équipements qui s'élève à 15 pour Abondant et à 19 pour Cherisy. Ce type de bourg n'est pas représenté dans les Yvelines. En effet si les deux premiers niveaux du réseau urbain se répartissent équitablement sur l'ensemble des deux départements, la base est en revanche plus dissymétrique. En Eure-et-Loir subsistent davantage de communes ayant moins de commerces et de services. Dans les Yvelines, cette catégorie est quasi inexistante. Un pôle de catégorie A ne survit comme tel qu'à partir de 20 équipements ou plus, compte tenu de la proximité et de la concurrence d'un centre urbain de rang supérieur et du fait que certains ménages fréquentent les commerces et services situés près de leur lieu de travail.

7 En dehors de Nogent-le-Roi situé bien au sud de la N12, et de Thoiry au Nord, tous les autres pôles cités ne sont guère éloignés de plus de $5 \mathrm{~km}$ de la voie express. Sur les 14 pôles du réseau urbain décrit, 5 sont directement sur l'axe N12 et 4 à moins de $2 \mathrm{~km}$. L'armature des centres de services étudiés s'ancre donc sur cette artère centrale qu'est la N12.

\section{Des centres aux fonctions renouvelées}

S'intéresser aux fonctions de ces pôles permet d'appréhender le renouvellement des pratiques en termes de déplacements quotidiens. L'enquête est l'outil indispensable pour saisir au mieux les mutations à l'œuvre. Elle permet également de réactualiser les données fournies par l'Inventaire communal.

9 L'accès à un commerce de base, comme l'alimentaire, apparaît comme une première entrée intéressante. Les déplacements qu'il nécessite étant fréquents, il renseigne sur les parcours empruntés et sur les choix faits par les ménages concernant le type de distributeur ${ }^{3}$. Le pôle dans lequel ils se rendent est donc bien identifiable. Une première 
tendance concerne les deux départements, les Yvelines et l'Eure-et-Loir. Elle pourrait paraître assez surprenante. Pour les trois quarts des personnes interrogées (cf. encadré 1), le supermarché est le premier distributeur fréquenté et non l'hypermarché. Un ménage enquêté sur trois seulement a cité les hypermarchés tels Auchan à Plaisir et Maurepas, puis Cora à Dreux. Pourtant Plaisir et Dreux se trouvent directement sur l'axe de la N12. Il s'agit d'habitants d'Eure-et-Loir. Ceux qui fréquentent en premier lieu ces très grandes surfaces ont un profil en effet différent selon les départements. Dans les Yvelines, ce sont des personnes qui, en général, habitent la commune depuis plus de vingt ans. Les nouveaux résidents, situés dans la tranche d'âge des 30-45 ans, sont ceux qui fréquentent le moins ce type de structure. Ceci peut être un nouveau choix de vie pour d'anciens citadins. En Eure-et-Loir, en revanche, ce sont plutôt les jeunes ménages qui s'y rendent. Ce peut être lié au fait qu'ils sont moins aisés que dans les Yvelines (Berger, 2004) : des différences de modes de vie et de comportements de mobilité en fonction des groupes sociaux et des trajectoires résidentielles ont été soulignées, par exemple, dans les couronnes périurbaines de Dijon (Sencébé, 2006) ou de Toulouse (Jaillet et al., 2006).

\section{ENCADRÉ 1}

Le terrain de l'étude ${ }^{4}$ comprend 96 communes, 55 dans les Yvelines et 41 en Eureet-Loir. La population est passée de 110267 habitants en 1990 à 123656 en 1999. Les deux tiers des communes ont moins de 1000 habitants et un tiers d'entre elles en ont moins de 400 . Une enquête a été réalisée auprès de ménages résidant dans quatre communes proches de la voie express, dans les Yvelines et en Eure-et-Loir. Le nombre d'enquêtés est de trente ménages dans chacun des deux départements. L'échantillon se compose de :

- vingt ménages récemment installés dans la commune (depuis moins de dix ans) : il s'agit de connaître les motivations de leur arrivée dans ce périmètre desservi par la nouvelle N12 et de comprendre l'usage qu'ils font de cette voie aménagée ;

- vingt ménages installés depuis plus de dix ans : l'intérêt est de voir si les habitudes de déplacement ont évolué avec les améliorations de la N12 et si les pôles de service fréquentés aujourd'hui sont les mêmes qu'il y a dix ans et plus ;

- vingt ménages de retraités disposant d'un véhicule quelle que soit la date d'emménagement sur la commune : cette catégorie doit permettre de voir s'il existe un enracinement des ménages dans cette région. Beaucoup d'actifs s'y sont installés pour des raisons professionnelles avec l'intention de regagner leur contrée d'origine au moment de leur départ en retraite. La fixation des populations joue aussi dans le fonctionnement d'un bassin de vie, du moins lui donne une réalité plus grande. En raison du profil démographique de notre société, les pratiques des personnes retraitées sont un paramètre d'importance à prendre en compte dans l'aménagement des territoires.

10 On pouvait faire l'hypothèse que l'ouverture de la déviation de Jouars-Pontchartrain aurait accentué l'attractivité de Plaisir, au moins pour les habitants des Yvelines. Il n'en est rien. En réalité nombreux sont ceux qui ont évoqué les distributeurs de Houdan et Maulette, appartenant plutôt à la catégorie « supermarché ». Ces supermarchés attirent 
des populations aussi bien d'Eure-et-Loir que des Yvelines. Pour les communes de Garancières et de Galluis, le supermarché de référence est celui de La Queue-lesYvelines, fréquenté pour la plupart deux à trois fois par semaine. Les ménages de ces deux communes sont pourtant très proches de Plaisir par la N12. Cette tendance observée dans l'enquête est confortée par le réaménage-ment du supermarché Champion de Villiers-Saint-Frédéric. Situé non loin d'une sortie de la N12, et pourtant plus proche encore de Plaisir que les précédents, ce magasin répond à une demande forte. Le projet d'extension et de restructuration de l'espace commercial dont il fait partie est le signe d'une fréquentation élevée. En revanche Plaisir semble acquérir de nouvelles fonctions. Longtemps associée à l'image de l'hypermarché Auchan, la commune se dote d'une série de grandes enseignes pour l'équipement de la maison. Elle tend ainsi à devenir un nouveau pôle commercial régional, à l'image de celui d'Orgeval et de Coignières. L'alimentaire n'est plus le premier motif de fréquentation de ce pôle urbain pour les habitants du secteur. Il a pourtant longtemps rempli de façon quasi exclusive cette fonction.

11 Pour un ménage sur trois, les supermarchés de Houdan, de Maulette ou celui de La Queue-les-Yvelines sont le seul lieu d'achat pour l'alimentaire. Pour les deux tiers restants, les supérettes et petits commerces sont des relais non négligeables. Dans les Yvelines, pour les communes enquêtées, La Queue-les-Yvelines est le premier bourg cité pour les petits commerces puis viennent Montfort-l'Amaury et Garancières. La place que tiennent les marchés dans ces déplacements offre des informations complémentaires sur ces nouvelles pratiques. Celui de La Queue-les-Yvelines est le plus souvent cité, puis vient celui de Galluis et enfin le marché de Montfort-l'Amaury. Ce sont des personnes assez jeunes qui, en venant s'installer dans le secteur, cherchent un style de vie allant de pair avec la consommation de produits de qualité, quitte à en payer le prix. En Eure-et-Loir, les bourgs fréquentés pour les petits commerces et supérettes sont avant tout Houdan, puis, plus au sud, Nogent-le-Roi. Au nord de la N12, Bû puis Abondant sont souvent évoqués. La moitié des ménages enquêtés dans ce département disent fréquenter un marché forain. Les marchés cités sont ceux de Dreux, le plus souvent, puis Houdan et plus rarement Ézy-sur-Eure. Les personnes concernées ont bien souvent plus de 50 ans. Leur profil est donc différent des ménages habitant les Yvelines.

On constate que les pôles les plus attractifs, pour leurs commerces alimentaires, ont un accès direct à la voie express. Ces commerces, qui ont pourtant depuis plusieurs années été évincés de nombreux bourgs par la concurrence des hypermarchés, ne seraient-ils pas en train de retrouver leur place? À travers les nouveaux modes de vie que recherchent les résidents apparaît la volonté de fuir le dense, l'urbain (Dubois-Taine, Chalas 1997). Les représentations liées au small is beautiful transparaissent dans l'évolution des pratiques. Elles témoignent d'un resserrement plus que d'un élargissement des territoires fréquentés.

13 Une autre entrée pour repérer les pôles fréquentés par les habitants du secteur est de s'intéresser à un service spécialisé. Connaître les localisations des deux médecins spécialistes les plus consultés est par exemple un moyen de mesurer l'attractivité de certaines communes et de repérer la qualité des services proposés. Les trois quarts des enquêtés des Yvelines se rendent uniquement dans des communes proches. Il s'agit dans l'ordre de Montfort-l'Amaury, Houdan, La Queue-les-Yvelines, Méré, Beynes, Garancières. Pour les habitants d'Eure-et-Loir, la quasi-totalité des enquêtés se rend 
dans des communes du secteur: les communes citées sont avant tout Houdan, puis Dreux, Bû, Montfort-l'Amaury, Nogent-le-Roi, Villemeux-sur-Eure. Cette tendance vaut uniquement pour les spécialistes visités le plus souvent, ceux qui le sont de façon exceptionnelle sont localisés plus souvent dans la commune de résidence antérieure ou bien dans un centre urbain régional tel Versailles, voire à Paris.

Plusieurs personnes disent avoir choisi des spécialistes plus près de chez elles au fil du temps. Cette tendance est nette dans les Yvelines. En revanche en Eure-et-Loir, avant que la voie express soit ouverte, l'éloignement de Paris et de sa proche banlieue était davantage ressenti. Les habitants de ces communes avaient pris l'habitude de consulter des spécialistes de Dreux ou de Houdan, rarement ceux situés en banlieue parisienne. Ainsi ceux qui vivent depuis plus de dix ans dans la commune enquêtée n'ont guère changé leurs habitudes. La voie express n'a fait que raccourcir leur temps de parcours et donc amélioré l'accessibilité à ces deux pôles. Dans les Yvelines, c'est au contraire pour cette catégorie de personnes que les destinations ont le plus changé. Un certain nombre d'entre elles disait se rendre dans des communes proches ou faisant partie aujourd'hui de la ville nouvelle de Saint-Quentin-en-Yvelines, comme Bois d'Arcy, Versailles, Trappes ou Montigny-le-Bretonneux. Ces communes sont aujourd'hui plus directement accessibles par la N12. Pourtant leur fréquentation dans le domaine médical n'a pas été renforcée. Au contraire, plusieurs enquêtés ont abandonné ces destinations pour venir consulter à Garancières, Montfort-l'Amaury ou Houdan. Chez les retraités, les changements sont moins fréquents. Dans cette tranche d'âge, le fait d'être suivi depuis plusieurs années par un médecin de confiance est peut-être un facteur important dans la permanence des destinations, alors que la tendance est à leur resserrement.

Les accessibilités nouvelles offertes par la voie express revêtent donc plusieurs formes. Elles se mesurent tout d'abord en terme de gain de temps par rapport à un trajet inchangé. Elles correspondent ensuite à la possibilité d'accéder pour une même durée de parcours à plusieurs pôles à la fois qui peuvent entrer en concurrence. Enfin, elles opèrent un nouveau classement des destinations choisies : celui-ci joue en faveur des pôles de proximité et parfois au détriment des pôles plus importants d'intérêt régional mais éloignés.

\section{Vers l'émergence de bassins de vie autour de Houdan et Montfort-l'Amaury}

Le bassin de vie peut être une clé pour comprendre l'organisation territoriale des espaces périurbains. L'Insee a en effet tenté de définir un nouvel espace pour décrire et étudier les conditions de vie des Français car, depuis un demi-siècle, les territoires administratifs traditionnels, communes et cantons, correspondent de moins en moins à des espaces fonctionnels (Julien, Pougnard, 2004).

Dans le but de définir un maillage géographique pertinent pour l'étude des conditions de vie, l'Insee a donc cherché à délimiter les plus petits territoires dans lesquels peuvent s'accomplir la majorité des actes quotidiens: l'accès aux services privés et publics fréquentés assez souvent et l'accès à l'emploi. Hors du milieu urbain dense, 1745 ensembles ont été délimités en France métropolitaine. Ces ensembles ont été nommés «bassins de vie des bourgs et petites villes». Parmi eux, certains sont considérés comme dépendants car ils n'ont pas un nombre suffisant d'équipements ou 
d'emplois pour répondre aux besoins de leurs habitants qui doivent donc plus souvent en sortir. La grande majorité des bassins de vie dépendants ont un pôle de nature périurbaine: ils sont dépendants des grandes agglomérations dont ils sont proches pour l'emploi et pour les services à la population. Pour autant cette dépendance n'est pas synonyme de déclin démographique.

Cette proposition de zonage par bassin de vie de l'Insee peut permettre d'appréhender l'organisation des espaces vécus autour de la N12. Les communes étudiées des Yvelines et de l'Eure-et-Loir sont précisément représentatives des marges de l'urbain dense avec une densité moyenne de $206 \mathrm{hab} / \mathrm{km}^{2}$ pour le canton de Monfortl'Amaury et de $97 \mathrm{hab} /$ $\mathrm{km}^{2}$ pour celui de Houdan. L'enquête apporte des informations complémentaires pour saisir ces bassins de vie et voir comment ils se combinent. En effet, l'Insee en dénombre quatre sur l'espace concerné : un en Eure-et-Loir, celui de Nogent-le-Roi, trois dans les Yvelines, autour de Houdan, de La Queue-les-Yvelines et de Montfort-l'Amaury. Or les bassins de La Queue-les-Yvelines et de Montfort-l'Amaury sont constitués par deux pôles qui sont très proches. N'y a-t-il pas alors chevauchement d'aires d'influences? L'essentiel est de savoir quel rôle structurant joue la voie express dans leur organisation.

une étude réalisée par l'Insee pour le Conseil général des Yvelines sur les dynamiques du tissu productif et leurs impacts en terme d'attractivité et d'emplois, la zone de Houdan-Montfort-l'Amaury apparaît bien comme une entité à part. Cet espace est présenté comme ayant une vocation agricole avec l'ensemble des plaines de Neauphle, d'Orgerus et du Drouais autour de Houdan. Le massif forestier de Rambouillet épouse assez bien les contours de la zone. Si le poids de ce territoire, en termes de population et d'emploi, reste modéré par rapport au total yvelinois, il ne faut pas omettre de comptabiliser les populations d'Eure-et-Loir qui entrent dans cette aire d'influence. Houdan et Montfort-l'Amaury, malgré leur taille modeste, ont su maintenir une attractivité auprès d'une part importante de la population de la zone.

L'historicité très marquée de ces chefs-lieux de canton participe à la légitimité de leur repositionnement comme places centrales. Or l'activité d'un centre-ville est basée, entre autres, sur la vitalité de ses commerces. Nombre d'entre eux sont soumis à la concurrence des grandes surfaces. Le commerce alimentaire est encore une fois une référence pour mesurer ce potentiel attractif. Dans ce secteur, le commerce de détail de pain, pâtisserie et confiserie est un bon indicateur. Il permet de mesurer les capacités du petit commerce à se maintenir. Il en est de même des supérettes. Entre 1993 et 2003, aucune radiation n'a été enregistrée par la Chambre de Commerce et d'Industrie pour ces deux types d'activités, que ce soit à Houdan ou à Montfort-l'Amaury. En revanche, plusieurs créations ${ }^{5}$ sont enregistrées. Houdan compte deux nouveaux magasins d'alimentation générale, ces dix dernières années. Durant cette même période, on enregistre aussi une création à Montfort-l'Amaury. Dotés de 32 équipements sur 36 par l'Inventaire communal, Houdan et Montfort-l'Amaury offrent un panel de services plutôt large dans les domaines suivants : le commerce, les équipements scolaires et de loisirs, les services administratifs et bancaires, la santé et l'aide à la personne. Chaque pôle a son propre hôpital. Ces hôpitaux ont essentiellement pour vocation de proposer des diagnostics et des séjours de convalescence. Les opérations chirurgicales se font ailleurs, en dehors du bassin. 
Carte 2. Réseau de transports et attractivités autour de la voie express.

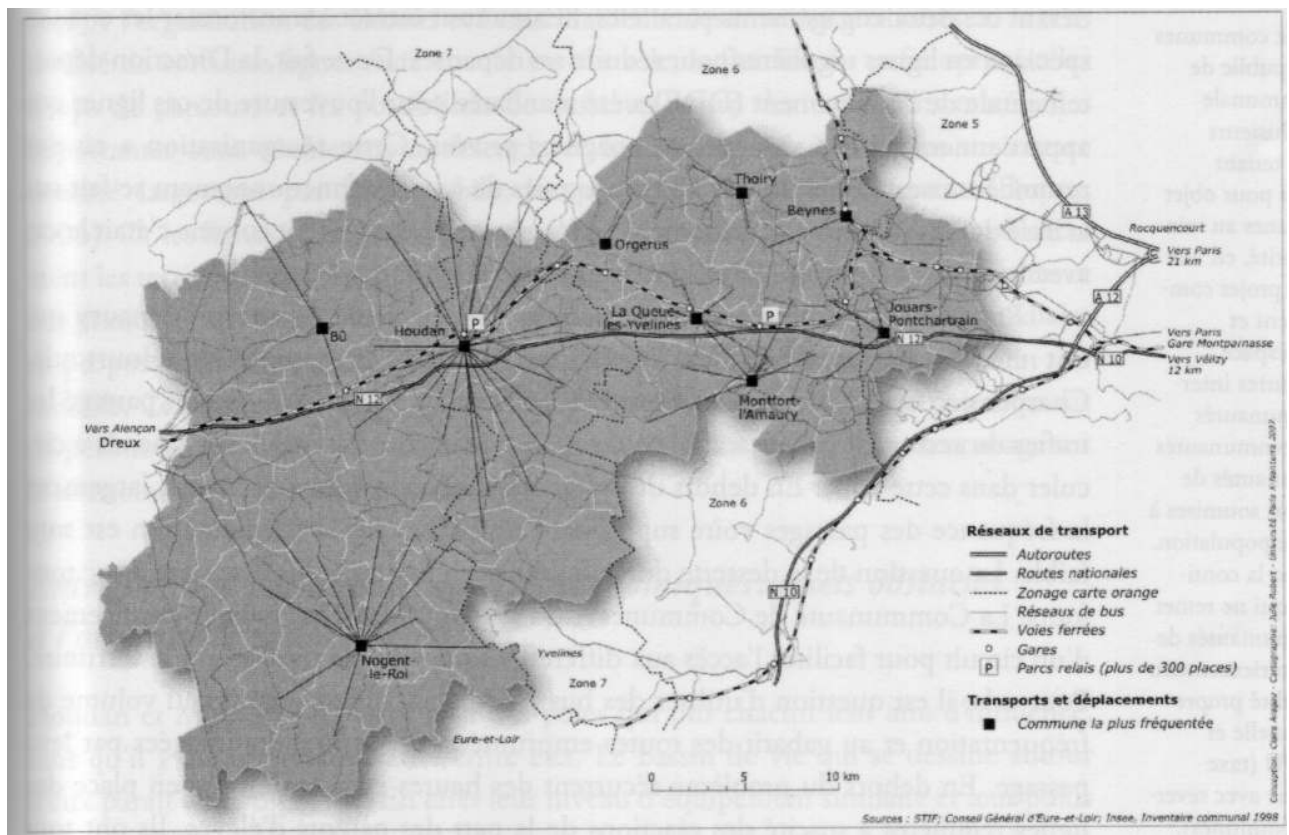

21 L'aire d'influence que ces deux centres organisent en terme de services courants est structurée par un réseau de communications qui s'appuie sur la voie express et dont les performances s'accentuent (carte 2). Houdan et Montfort-l'Amaury voient leurs positions de carrefour se renforcer mutuellement. À proximité de leurs gares, des parcs relais ont été réalisés, comptant chacun plus de 300 places de parking. Ces gares sont le point de passage de lignes régulières de bus qui desservent les communes voisines. La distinction entre circuits spéciaux et lignes régulières est importante car elle touche à la question de l'accessibilité de certains lieux. Les circuits spéciaux dépendent d'un Sivom ${ }^{6}$ ou d'une communauté de communes et sont donc sous l'autorité des communes qui en font partie. Concernant la desserte des établissements scolaires, l'objectif du Sivom se limite à la bonne gestion du ramassage scolaire pour le secteur qui l'intéresse. L'État et le Conseil général participent financièrement à la mise en place de ces circuits, mais les communes restent bien l'autorité organisatrice. Progressivement les circuits spéciaux tendent à être transformés en lignes régulières. En plus des établissements scolaires, la ligne régulière est destinée à tout type d'usager dans la mesure où elle doit marquer des arrêts aux gares SNCF et aux centres commerciaux. Elle vise donc à désenclaver des communes qui étaient jusque-là véritablement captives, privées de transport. Si l'État apporte un soutien financier aux circuits spéciaux, il participe aussi au financement de la carte Imagine $\mathrm{R}^{7}$. En considérant ces deux engagements parallèles, l'État a tout intérêt à transformer les circuits spéciaux en lignes régulières pour réduire ses dépenses. De ce fait, la Direction départementale de l'équipement (DDE) a été mandatée pour l'ouverture de ces lignes qui appartiennent désormais à des compagnies privées. Cette réorganisation a eu des retombées controversées pour les compagnies de cars. Le fonctionnement se fait sur la base du nombre de voyageurs transportés et non plus au forfait comme c'était le cas avec les circuits spéciaux. La sécurité financière n'est donc plus assurée.

Prenons l'exemple des circuits spéciaux du Sivom de Montfort-l'Amaury qui ont été intégrés à des lignes régulières. Les trois transporteurs, Hourtoule, Charpentier/CGEA Houdan et Connexe Centre de Rambouillet, se sont partagé les trafics du secteur. Aux 
heures de pointes, 21 à 22 cars Hourtoule sont amenés à circuler dans cette zone. En dehors de ces heures, les compagnies réduisent largement la fréquence des passages voire suppriment des lignes car la fréquentation est trop faible. La question de la desserte des communes en heures creuses se pose donc toujours. La Communauté de Communes $^{8}$ du Pays Houdanais envisage le financement d'un circuit pour faciliter l'accès aux différents équipements répartis sur le territoire. Pour cela, il est question d'utiliser des bus plus petits, mieux adaptés au volume de fréquentation et au gabarit des routes empruntées, souvent endommagées par leur passage. En dehors du problème récurrent des heures creuses, la mise en place des lignes régulières a suscité des réactions de la part des parents d'élèves. Ils ont tout d'abord été inquiets pour la sécurité de leurs enfants à l'intérieur des cars en raison de l'ouverture de la ligne à des personnes autres que des scolaires. Ensuite la cherté du billet de transport (carte Imagine $\mathrm{R}$ ou carte Optile ${ }^{9}$ a suscité des mécontentements. Mais le Conseil Général a fini par proposer une compensation pour les titulaires de la carte Imagine R. Rendre des espaces accessibles demande des compromis et une ouverture aux risques venus de l'extérieur.

Le gain de temps apporté par la nouvelle voie est en réalité moins lié à la possibilité d'emprunter la N12 qu'au fait que la traversée de certaines communes est désormais facilitée. L'exemple cité en premier lieu par les cars Hourtoule, dont le dépôt se trouve à Plaisir-les-Gâtines (à l'ouest du secteur d'étude), est la traversée de JouarsPontchartrain réalisée en moins de trois minutes depuis l'ouverture de la déviation. Pour de nombreuses lignes, le passage du nord au sud de la N12 se fait par Pontchartrain. C'est le cas de la ligne 078 Mantes-Saint-Quentin-en-Yvelines. L'utilisation de la voie express est tout de même intéressante pour les cars qui partent à vide du dépôt et se rendent au point de départ de leur ligne. Le gain de temps est réel là encore à la hauteur de Pontchartrain, mais aussi à la sortie ouest de la commune, au Pontel, autre goulet d'étranglement auparavant. La déviation permet de surmonter ces deux obstacles. Le temps économisé sur ces parcours peut être réutilisé autrement. Il permet notamment de renouveler les passages sur certaines lignes, ce qui était compromis auparavant. La voie express a donc bien participé à l'évolution de l'organisation des circuits et à une meilleure desserte des territoires. Si la rentabilité de certaines lignes reste encore un obstacle, la fluidité du trafic optimise les temps de parcours et les possibilités de dessertes. Ainsi des conditions matérielles importantes sont réunies pour désenclaver les communes. Mais dans un espace dominé largement par l'automobile, les pratiques évoluent lentement (Dupuy, 2006). Or les lignes régulières n'attendent que leurs passagers pour irriguer efficacement les territoires traversés. En cette période marquée par l'évolution des attentes et des pratiques, les communautés de communes peuvent jouer un rôle intermédiaire en proposant la desserte des équipements partagés pour renforcer leur cohésion territoriale. Cette première étape dans le développement des transports en commun sur un périmètre limité peut se présenter comme un tremplin pour étendre leur usage sur le territoire plus vaste du bassin de vie. 


\section{Entre volonté politique et réalités économiques : quels obstacles à l'organisation des territoires ?}

Houdan et Monfort-l'Amaury sont des pôles qui ont chacun leur aire d'influence, sans qu'il y ait complémentarité entre eux. Le bassin de vie qui se dessine autour d'eux paraît donc bipolaire. En effet leur niveau d'équipement similaire et leur poids démographique ne privilégient aucun d'eux. Le profil de leurs aires de chalandise est bien distinct. Celui de Houdan est rural avant tout puis résidentiel. Pour Montfortl'Amaury, c'est l'inverse: le caractère résidentiel prime sur l'aspect rural. On peut penser que c'est leur potentiel de développement respectif qui donnera à l'un plutôt qu'à l'autre un plus grand rayonnement.

Pour devenir un pôle structurant sur un territoire où la taille moyenne des communes est de 300 habitants, la masse critique est de l'ordre de 5000 habitants. Montfortl'Amaury (3 137 habitants en 1999) associé à Méré, la commune limitrophe (1 675 habitants en 1999) qui accueille la gare portant les deux noms, atteint ce seuil. Mais si cette association remplit les conditions pour constituer une seule entité, des arrièrepensées politiques viennent freiner leur fusion. Le journal Les Nouvelles de Rambouillet affirme : « Méré ne sera jamais le satellite de Montfort ». Pourtant si ce n'était pas le cas autrefois, aujourd'hui, ces deux communes sont très complémentaires. Montfortl'Amaury a une forte identité patrimoniale, détient l'essentiel de l'équipement commercial et des activités de service (écoles, poste, hôpital). Méré compte une zone d'activité de 24 ha ainsi que la gare et son parc relais, nœud de communication permettant les échanges entre les communes situées au nord et au sud de la voie express. Montfort-l'Amaury commence à pâtir du retard de la mise en place d'une communauté de communes dont elle serait le cœur. L'ouverture de la voie express a accéléré le processus de réflexion. Il est urgent d'assurer un développement économique le long de la N12 entre les communes de Galluis, La Queue-les-Yvelines et même Garancières. Ce développement doit être distinct de celui de Houdan pour conserver une identité montfortoise mais aussi distinct de Jouars-Pontchartrain, Neauphle-le-Château et Villers-Saint-Frédéric, qui ont rejoint la Communauté de Communes "Cœur d'Yvelines" dont le centre de gravité se trouve au nord de la voie express. Se constituer en communauté de communes permet de faire face aux risques liés à une démographie galopante et incontrôlée: populations supplémentaires et demandes accrues de terrains à bâtir viennent rompre l'équilibre en termes de services offerts à la population. L'aménagement de la N12 a favorisé une prise de conscience car jusque-là la nécessité économique de se constituer en communauté de communes ne se faisait pas sentir. Mais c'est aussi le contexte politicoadministratif qui va dans ce sens. À l'heure actuelle, la Communauté de Communes du «pays montfortois » est sur le point de voir le jour.

En revanche, Houdan (3 112 habitants en 1999) a été très tôt désigné dans les documents d'aménagement comme un pôle rural de développement à l'entrée de l'îlede-France. Avec Maulette (712 habitants en 1999), ce pôle a vocation à compter 5000 à 6000 habitants. En raison d'un contexte économique différent de celui de la région Îlede-France, des communes d'Eure-et-Loir ont éprouvé le besoin de faire partie de la Communauté de Communes du Pays Houdanais (CCPH) créée le $1^{\mathrm{er}}$ janvier 1998, qui compte aujourd'hui 32 communes membres. Vingt-sept d'entre elles sont situées dans les Yvelines et cinq en Eure-et-Loir, ce qui démontre une volonté forte de bâtir un 
projet commun transcendant les frontières administratives. La CCPH sera la première bénéficiaire du nouveau contrat de développement équilibré mis en place par le département. Ce contrat concerne des territoires qui présentent une masse critique suffisante en termes d'emploi et de population. Les communes doivent conjuguer un certain nombre d'atouts en termes de développement urbain, de développement économique et d'attractivité du cadre naturel et urbain. Ainsi le potentiel de développement de Houdan est affiché comme supérieur à celui de Montfort-l'Amaury. Sa situation aux marges de l'île-de-France est un facteur décisif dans sa désignation comme pôle structurant.

Qu'en est-il de la réalité économique de ce bassin Houdan-Montfort-l'Amaury, territoire dont l'Insee et le département reconnaissent l'émergence ? Ce bassin de vie remplit-il les conditions nécessaires en terme d'offre d'emploi ? Les navettes domiciletravail révèlent qu'un tiers seulement des résidents a un emploi dans le bassin HoudanMontfort-l'Amaury. Ce bassin de vie est donc largement dépendant des pôles d'emploi situés essentiellement dans la grande couronne parisienne incluant la ville nouvelle de Saint-Quentin-en-Yvelines. Mais il l'est aussi, dans une proportion non négligeable, d'un espace plus lointain comprenant des lieux de travail en province ( $29 \%$ des actifs ont un emploi hors île-de-France). Pour autant cette dépendance n'est pas synonyme de déclin démographique, comme l'atteste une croissance de la population de $+1,3 \%$ en moyenne par an entre 1990 et 1999. En outre, la création de huit nouvelles zones d'activité sur ce territoire depuis 1990 témoigne de ses potentialités de développement en matière économique. Ces réalisations interviennent après des études de faisabilité qui enregistrent une demande. Cela sans oublier le projet à l'œuvre concernant la création d'une zone d'activité de 40 ha, sous la maîtrise d'ouvrage de la Communauté de Communes des villages du Drouais, sur la commune de Germainville, là encore le long de la N12.

\section{Conclusion}

L'analyse du rôle des pôles secondaires de Houdan et de Montfort-l'Amaury met donc en évidence l'évolution des pratiques de déplacements des habitants et les représentations qu'ils ont des territoires qu'ils parcourent. Elle montre qu'en dépit de la proximité et du poids de la métropole parisienne ainsi que de ses pôles d'emploi, l'organisation des territoires quotidiens des habitants des marges périurbaines de l'îlede-France s'appuie sur un réseau de petites villes et de bourgs centres dont le rôle se renforce quand leur accessibilité s'améliore. Loin de signer leur déclin au profit de pôles mieux dotés en équipements, la création d'une voie rapide renforce leurs fonctions de desserte d'une clientèle locale en quête d'un meilleur ancrage dans un bassin de vie à échelle plus humaine. Les périurbains voudraient de la proximité et constituent au fil du temps des territoires de proximité, comme l'ont montré, par exemple, des travaux sur les mobilités des habitants de la Seine-et-Marne (Aguilera et al., 2006). Les espaces périurbains ne sont plus seulement des espaces dortoirs, une attractivité, des polarisations se mettent en place. Les pouvoirs publics locaux œuvrent aussi à la constitution de ces territoires de proximité à travers la réalisation de communautés de communes et l'organisation de circuits de transport en leur sein. Demande et offre de « territorialisation » se rencontrent. 


\section{BIBLIOGRAPHIE}

Aguilera A., Massot M.-H., Proulhac L., 2006, « L'intégration du périurbain francilien à la métropole parisienne. Une mesure des flux quotidiens de personnes ", in Larceneux A., BoiteuxOrain C. (dir.), Paris et ses franges : étalement urbain et polycentrisme, Dijon, Éditions universitaires de Dijon, p. 73-97.

Berger M., 2004, Les périurbains de Paris. De la ville dense à la métropole éclatée ? Paris, CNRS Éditions, $317 \mathrm{p}$.

Cohou M., 2000, Le destin d'une voie rapide, jeu des acteurs, enjeu de développement, Toulouse, Presses universitaires du Mirail, $165 \mathrm{p}$.

Dubois-Taine G., Chalas Y., 1997, La ville émergente, La Tour d'Aigues, Éditions de l'Aube, 285 p.

Dupuy G., 2006, La dépendance à l'égard de l'automobile, Paris, La Documentation française, PREDIT (Programme de recherche et d'innovation dans les transports terrestres), $96 \mathrm{p}$.

Jaillet M.-C., Rougé L., Thouzellier C., 2006, « Modes de vie des navetteurs toulousains installés aux franges de l'aire urbaine ", in Bonnet M., Aubertel P. (dir.), La ville aux limites de la mobilité, Paris, PUF, p. 161-173.

Julien P., Pougnard J., 2004, « Les bassins de vie, au cœur de la vie des bourgs et petites villes », Insee Première, $\mathrm{n}^{\circ}$ 953-avril.

Offner J.-M., 1993, « Les effets structurants du transport : mythe politique, mystification scientifique ", L'Espace géographique, 3, p. 233-242.

Ollivro J., 1998, « 15 minutes ou 1/4 d'heure ? Réalité et interprétation des grands projets infrastructurels », Transports, $n^{\circ} 388$, mars-avril, p. 108-111.

Ollivro J., 2000, L'Homme à toutes vitesses, de la lenteur homogène à la rapidité différenciée, Rennes, Presses universitaires de Rennes, février, $179 \mathrm{p}$.

Sencébé Y., 2006, « Mobilités quotidiennes et ancrages périurbains, attrait pour la campagne ou retrait de la ville ? », in Bonnet M., Aubertel P. (dir.), La ville aux limites de la mobilité, Paris, PUF, p. $153-160$.

Société des autoroutes Paris-Rhin-Rhône, 1994, L'impact économique de l'autoroute A71 BourgesClermont-Ferrand. Conclusion des travaux menés par l'Observatoire des effets économiques de l'autoroute A71 1986-1993, 31 p.

\section{NOTES}

1. Une Déclaration d'utilité publique est une procédure administrative qui permet de réaliser une opération d'aménagement, telle que la création d'une infrastructure de communication par exemple, sur des terrains privés en les expropriant, précisément pour cause d'utilité publique ; elle est obtenue à l'issue d'une enquête d'utilité publique.

2. Voir Inventaire communal de l'Insee, 1998. La catégorie A de l'Insee correspond au meilleur niveau de classement pour les équipements essentiels : "Commune disposant simultanément d'une part, soit d'un supermarché ou d'un hypermarché, soit à défaut, à la fois d'une boulangerie, d'une boucherie ou d'une charcuterie, d'un magasin d'alimentation générale ou d'une supérette, et d'un magasin de droguerie, quincaillerie, outillage ou d'une grande surface non alimentaire, 
et, d'autre part, d'un café ou d'un restaurant, d'un point de vente de quotidiens, d'un bureau de tabac et d'un bureau de poste ou d'une agence postale. »

3. On entend par distributeurs, les magasins dans lesquels sont vendus les produits alimentaires, aussi bien les petits commerces de détail que les très grandes surfaces de type hypermarché.

4. Cette étude a été réalisée dans le cadre d'une thèse de doctorat (thèse en cours, sous la direction de J.-P. Charvet, « Aménagement d'une voie express et renouvellement périurbain : le cas de la N12 »).

5. Derrière le terme de création se cache également la possibilité qu'il y ait simple reprise de l'activité par un autre propriétaire. Un changement de propriétaire induit pour la CCI une création. Cette information permet quoi qu'il en soit de mesurer la disparition ou au contraire le maintien des petits commerces en centre-ville.

6. Le Syndicat intercommunal à vocations multiples (Sivom) exerce des responsabilités variées (collecte et traitement des ordures ménagères, création et entretien de voirie, équipements sportifs, action sociale, transport etc.) qui lui ont été transférées par les différentes communes.

7. La carte Imagine R s'adresse aux jeunes de moins de 26 ans qui sont étudiants, ou collégiens, lycéens, ou apprentis en formation par alternance d'un niveau inférieur au baccalauréat. Cette carte est un abonnement valable un an pour voyager sur tous les transports d'île-de-France à un tarif préférentiel.

8. La communauté de communes est un établissement public de coopération intercommunale (EPCI) regroupant plusieurs communes d'un seul tenant et sans enclave. Elle a pour objet d'associer des communes au sein d'un espace de solidarité, en vue de l'élaboration d'un projet commun de développement et d'aménagement de l'espace. Contrairement aux autres intercommunalités (communautés d'agglomération et communautés urbaines), les communautés de communes ne sont pas soumises à un seuil minimum de population. La seule contrainte est la continuité géographique, qui ne remet pas en cause les communautés de communes créées antérieurement. C'est un EPCI à fiscalité propre par la taxe professionnelle et le plus souvent la TPU (taxe professionnelle unique avec reversement partiel aux communes).

9. La carte Optile est utilisable exclusivement en période scolaire. Elle permet à l'élève d'effectuer les trajets domicile-école. Un seul aller-retour quotidien est autorisé. Les tarifs dépendent du lieu de domicile de l'élève et du lieu de son établissement scolaire.

\section{RÉSUMÉS}

L'article analyse les effets du réaménagement récent en voie express de la N12, entre Bois-d'Arcy et Dreux. A-t-il accentué la polarisation du territoire par la ville nouvelle de Saint-Quentin-enYvelines et entraîné le déclin des petites villes et des bourgs situés le long ou à proximité de l'axe, tels Houdan ou Montfort-l'Amaury? À partir d'une enquête réalisée auprès de 60 ménages, il met en évidence l'évolution des pratiques de déplacements des habitants. En dépit de la proximité et du poids de la métropole parisienne ainsi que de ses pôles d'emploi, l'organisation des territoires quotidiens des habitants des marges périurbaines de l'île-de-France s'appuie sur un réseau de petites villes et de bourgs centres dont le rôle se renforce quand leur accessibilité s'améliore. Loin de signer leur déclin au profit de pôles mieux dotés en équipements, la création d'une voie rapide renforce leurs fonctions de desserte d'une clientèle locale en quête d'un meilleur ancrage dans un bassin de vie à échelle plus humaine. Les pouvoirs locaux accompagnent l'émergence de 
ces territoires de proximité aussi bien par l'organisation de circuits de transport public collectif qu'à travers la constitution de communautés de communes.

The article analyses the effects caused by the recent redevelopment along the N12 expressway between Bois-d'Arcy and Dreux. Has it further polarized the territory around the new town of Saint-Quentin-en-Yvelines and caused the decline of small towns and villages located along or close to the main road, such as Houdan or Montfort-l'Amaury? A survey of 60 households highlights how local people changed their travel practices. Despite the proximity and weight of the Parisian metropolis and its employment poles, the organization of daily territories of people living in the outer suburbs of the Ile-de-France region relies on a network of small towns and villages which play a more important role as they become more accessible. Far from signaling their decline to the benefit of better-equipped poles, the creation of an expressway reinforces their servicing role for local people who look for a better anchorage point in a more humane living basin. Local authorities sustain these emerging neighbourhood territories by organizing public transport systems and building up town communities.

\section{INDEX}

Mots-clés : quotidien, territoire, aménagement, transports, espace périurbain Index géographique : France

\section{AUTEUR}

\section{CLAIRE ARAGAU}

Professeur agrégée de géographie,

IUFM de Paris, Gecko (Laboratoire de géographie comparée des Suds et des Nords),

Université Paris X Nanterre,

200 avenue de la République,

92001 - Nanterre Cedex

claire.aragau@free.fr 